\title{
Arbeitsgestaltung als Informationsmanagement
}

\section{Ermitteln des Informationsbedarfs und Gestalten des Informationsflusses}

\author{
Winfried Hacker ${ }^{1}$ \\ Online publiziert: 22 . September 2020 \\ (c) Der/die Autor(en) 2020
}

\section{Zusammenfassung}

Mit dem Verbreiten vernetzter digitaler Arbeitsmittel in der Erwerbsarbeit wächst die Häufigkeit der Klagen über Informationsüberlastung, die mit Leistungs- und Gesundheitsbeeinträchtigungen einhergehen kann. Zur Vermeidung ist das systematische präventive Gestalten des Informationsflusses erforderlich. Dazu gehören: Unterscheiden zwischen der für die Auftragserfüllung erforderlichen Information und auftragsirrelevanten, störenden Daten; Anbieten der erforderlichen Information in einer für die Handlungsregulation geeigneten Darstellung; eine Arbeitsorganisation (insbes. Arbeitsteilung) sowie Medienwahl, die den erforderlichen Informationsfluss verringern; sowie das Auswählen bzw. Entwickeln informationstechnischer Systeme, die anforderungsgerechte, ,gebrauchstaugliche“ Information vermitteln. Hilfsmittel dafür werden skizziert.

Praktische Relevanz: Informationstechnologien nehmen sowohl im Büro- als auch im Produktionsbereich beständig zu. Diese Tatsache erfordert eine menschengerechte und ergonomische Gestaltung des Informationsmanagements, wozu dieser Artikel einen Beitrag leistet.

Schlüsselwörter Informationsüberlastung · Gestaltung des Informationsflusses · Gebrauchstauglichkeit · Arbeitsgestaltung als Informationsmanagement · Digitale Transformation

\section{Information management}

Identification of required information and design of information flow

\begin{abstract}
With the dissemination of interlinked digital means in gainful work, the frequency of complaints on information overload increases and is often connected with impairments of employee's performance and health. For prevention, the systematic design of information flow is necessary. This includes: the differentiation between information necessary to fulfill the work order versus task-irrelevant data; the offer of the required information in an action-regulating quality; the reduction of excessive information flow by suitable work organization (e.g. division of labour) and a considered type of information media as well as the selection of human-centered interactive systems. Means for these purposes are offered.
\end{abstract}

Keywords Information overload - Design of information flow $\cdot$ Human-centered design and selection of interactive systems $\cdot$ Information management $\cdot$ Digital transformation

Winfried Hacker

winfried.hacker@tu-dresden.de

1 Fakultät Psychologie, TU Dresden, 01062 Dresden, Deutschland 


\section{Quellen und Folgen von Informationsüberlastung in der digitalen Erwerbsarbeit - Viele Daten, wenig Information?}

Mit dem Verbreiten vernetzter digitaler Arbeitsmittel und der E-Mail-Kommunikation wuchs die Häufigkeit von Klagen über Informationsüberlastung bzw. Zeitdruck bei der Informationsverarbeitung: Viel bzw. zu viel Information sei in der verfügbaren Zeit zu bearbeiten (Drössler et al. 2018). Die Bezeichnung als Informationsüberlastung ist unzutreffend und irreführend: Häufig liegen zwar zu viele auftragsirrelevante Daten vor, aber tätigkeitsleitende Information kann gleichzeitig fehlen. Verzögerungen durch das Beschaffen fehlender Information sind eine der Ursachen für sozial und/oder wirtschaftlich belastende Unterbrechungen im Informationsfluss (z.B. Baethge und Rigotti 2013; Hacker 2020).

Das Erleben von Informationsüberlastung kann mehrere Quellen haben:

a. Die Arbeitenden werden durch Sachverhalte behelligt, die mit dem Ausführen ihres bearbeiteten Arbeitsauftrags nichts zu tun haben, also unterbrechen und stören und dabei Zusatzaufwand erzeugen (Baethge und Rigotti 2013; Lin et al. 2013; Mark et al. 2008).

$\mathrm{Zu}$ diesen störenden Meldungen gehören z.B. überflüssige CC-Setzungen lediglich zur Absicherung, zum Erzeugen des Eindrucks von Aktivität, Kommunikationen, die durch fehlende Vertretungsregelungen, unklare oder übertriebene Arbeitsteilung erzeugt werden (Mark 2002) oder Spam.

Störungen liegen auch dann vor, wenn derartige Meldungen nicht gelesen werden, sondern nur ihr Eingang reflektorisch registriert wird (Hassin 2014).

b. Nicht prinzipiell auszuschließen ist, dass Arbeitende zur Informationsüberlastung selbst beitragen aufgrund der Verführung zu privater Kommunikation während der Arbeitszeit durch digitale Technik.

c. Eine (zu) hohe Menge informationsüberarbeitender Arbeitsaufträge für die verfügbare Zeit.

Abhilfe hierbei kann die anforderungsgerechte Bemessung der Auftragsmenge für die verfügbare Zeit bzw. der verfügbaren Zeit für die geforderte Menge (Zeit- und Personalbemessung) schaffen (Rau und Göllner 2018).

d. Ein (zu) hoher Bearbeitungsaufwand durch die inhaltlichen und organisatorischen Anforderungen des informationsverarbeitenden Arbeitsprozesses, insbesondere durch

1. die Anzahl gleichzeitig zu berücksichtigender Merkmale je Arbeitsschritt, welche die begrenzte menschliche Verarbeitungskapazität übersteigt und daher zusätzliches Behalten (Merken) und Wiederaufnehmen von Überlegungen mit zusätzlichem Zeitbedarf erzeugt (Cowan 2010).

2. Mangelhafte Gebrauchstauglichkeit der Information, die Zusatzaufwand mit zusätzlichem Zeitbedarf erfordert (vgl. Abschn. 4).

3. Verbesserungsbedürftige Arbeitsorganisation, die zusätzlichen Aufwand und Zeitbedarf erzeugt u. a. durch Störungen/Ablenkungen/Unterbrechungen (z. B. durch E-Mails), die zusätzliches Behalten des unterbrochenen Arbeitsschrittes und Wiedereinarbeitung erfordern (Baethge und Rigotti 2013; Drössler et al. 2018).

Der Zusatzaufwand bei d.2 und d.3 belastet die begrenzte Verarbeitungskapazität (d.1) und verschlechtert dabei auch die Einprägungsaktivitäten und das Behalten für ein späteres Wiederaufgreifen. Damit entsteht ein Teufelskreis.

Informationsüberlastung hat sowohl soziale als auch wirtschaftliche Folgen:

Das Wohlbefinden der Arbeitenden kann durch die wahrgenommene Überforderung beeinträchtigt sein (Junghanns und Kersten 2020). Mittelfristig kann das beeinträchtigte Wohlbefinden Gesundheitsbeeinträchtigungen zur Folge haben, die zu Arbeitsbefreiungen führen können.

Im Zusammenhang damit können die Arbeitsleistungen - in Abhängigkeit von der Anforderungsart - in Qualität und/oder Menge beeinträchtigt sein (Seidler et al. 2018).

Abhilfe gegen das Überlasten der begrenzten Verarbeitungskapazität kann insbesondere das Unterscheiden zwischen tatsächlichem Informationsbedarf und Daten im Informationsmanagement schaffen (vgl. Abschn. 3); in einzelnen Fällen gegebenenfalls auch das Zusammenfassen mehrerer Einzelmerkmale zu einem Komplexmerkmal.

Abhilfe bei mangelhafter Gebrauchstauglichkeit der tatsächlich benötigten Information schafft das Sichern gebrauchstauglicher Information durch handlungsgerechte Informationsgestaltung (vgl. Abschn. 5).

Abhilfe bei verbesserungsbedürftiger Arbeitsorganisation als Quelle der Informationsüberlastung ist durch überlegte Wahl des Informationsmediums anstelle der Digitalisierung um jeden Preis (Abschn. 6.3) sowie durch daten- (nicht informations-) reduzierende und störungs-/ unterbrechungsfreie Arbeitsorganisation (Abschn. 6.2) zu schaffen.

\section{Informationsmangel trotz Datenflut - Mögliche objektive und subjektive Gründe}

Trotz vieler eintreffender Daten kann die erforderliche Information für das sachgerechte Ausführen einer Arbeitstätigkeit oder für das Fällen von Entscheidungen fehlen.

Abgesehen von objektiv fehlender, zunächst zu ermittelnder, zu recherchierender oder gar zu erforschender In- 
formation, kann Information im Arbeitsprozess fehlen aufgrund von Mängeln im inner- oder zwischenbetrieblichen Fluss der benötigten Information.

Diese Mängel gehen häufig zurück auf subjektive Unklarheiten, wer welche Information wem wann zu geben hat (Bringe-Information), und wer welche Information von wem zu erfragen hat (Hole-Information).

Hierbei kann ein Kommunikations-Dilemma entlang einer Wertschöpfungskette vorliegen, dem das betriebliche Informationsmanagement vorbeugen müsste:

Der Informationsgeber eines nachgeordnet arbeitenden Informationsnehmers hat individuelle Annahmen, welche Information dieser wann benötigt (was er/sie wissen muss), sowie welche Information er bereits besitzt (was er/sie weiß), sowie welche Information er geben muss (BringeInformation) und welche der andere sich holen wird/sollte (Hole-Information).

Eine Pflegekraft bemerkt, dass ein Patient die ausgegebene Schlaftablette im Nachttisch verwahrt und bittet ihn, er möge das Einnehmen nicht vergessen. Ihre Kollegen oder die Pflegedienstleitung informiert sie über ihre Beobachtung nicht.

Später versucht der Patient mit den gesammelten Tabletten einen Suizid.

Außerdem sieht man die Arbeitsanforderungen anderer durch die „Brille“ der eigenen Anforderungen. Bei artteiliger Arbeitsteilung können damit unterschiedliche Meinungen über den objektiven Informationsbedarf eines Arbeitsplatzes entstehen (z. B. Hacker 2008, Teil III).

Das Ermitteln bestehender Informationsfluss-Mängel und der Verletzung von Hol- und Bringepflichten von Informationen allein durch Befragen wäre heikel: Wenn ein Erwerbstätiger nicht realisiert, dass er über eine Information verfügt, die ein anderer benötigt, ist es schwierig, dies von ihm zu erfragen.

Das Auswerten dokumentierter Daten (DokumentenAnalyse) kann Verbesserungsbedarf im Informationsfluss erkennbar machen (z.B. dokumentierte Reklamationen, Lieferirrtümer, Nacharbeit, Stillstandszeiten wegen fehlender Informationen; z. B. Hacker 2008, Abschn. 3.4). Vertiefend sind Beobachtungsinterviews (Kuhlmann 2002) unerlässlich.

Durch systematisches Verbessern des Informationsflusses zwischen Kunden, Konstruktion und Fertigung in einem Kleinbetrieb des Sondermaschinenbaus wurden die Mängelrate in der Konstruktion um 3,5\%, die Nacharbeitskosten um $8 \%$ gesenkt sowie die Produktivität im CNC-Zentrum um fast $50 \%$ gesteigert (Fuchs, Pietzcker in Hacker 2008, S. 59).

\section{Die Hauptfrage: Information oder nur Daten?}

Der umgangssprachliche Informationsbegriff eignet sich nicht für das betriebliche Informationsmanagement. Er kann zum Erleben von Informationsüberlastung beitragen und ein rationelles Informationsmanagement behindern.

Im Erwerbsarbeitsprozess wird zur Auftragserfüllung Information im informationswissenschaftlichen Verständnis benötigt. Das sind Sachverhalte, die bestehende Unklarheiten (Nichtwissen) beseitigen, im Erwerbsprozess sind das Unklarheiten der Bearbeiter bezüglich ihrer zu bearbeitenden Arbeitsaufträge und der Ausführungsbedingungen und von ihnen zu treffenden Entscheidungen.

Was keine diesbezüglich bestehende Unklarheit beseitigt, ist keine handlungsleitende Information. Daher gibt es nicht zu viel Information, die zu viel handlungsbezogene Unsicherheit beseitigt, aber sehr wohl zu viele Daten (Attneave 1965).

Das Zuführen, Anbieten oder Beschaffen und Bearbeiten auftragsirrelevanter Daten besetzt unnütz die begrenzte mentale Verarbeitungskapazität der Arbeitenden und stört dadurch den Arbeitsprozess.

Praktische Schlussfolgerung:

Die Grundlage betrieblichen Informationsmanagements ist

- das Unterscheiden von Information als Beseitigung von Nichtwissen bezüglich der Arbeitsaufträge im informationswissenschaftlichen Sinn von Daten als aufgabenirrelevanten Meldungen. Zum Ermitteln des Bedarfs an Information i.e. S. kann ein Hilfsmittel dienen (Abschn. 4);

- das Abschirmen der Arbeitenden bei der Erwerbstätigkeit von überflüssigen Daten. Verbreitete Lösungen hierzu sind z.B. Spam-Filter in digitalen Arbeitsmitteln oder organisatorische Vorkehrungen gegen unnötige CC-Setzungen in E-Mails.

Beim Erwägen des Abschirmens von überflüssigen und beeinträchtigenden Auseinandersetzungen mit Daten ist zu beachten, dass die Beeinträchtigung nicht beschränkt ist auf das bewusste Aufnehmen: Daten - z. B. Gespräche im Hintergrund, das Anzeigen einer E-Mail - lenken auch reflektorisch die Aufmerksamkeit ab, besetzen die begrenzte Verarbeitungskapazität und stören den Arbeitsprozess.

Das Nutzen vernetzter digitaler Arbeitsmittel vervielfacht die Datenflut - umgangssprachlich Informationsflut - durch Vernetzungen über Raum und Zeit. Erforderlich ist also ein Informationsmanagement zum Verhüten der Datenflut. 


\section{Ermitteln des auftragsbedingten Informationsbedarfs}

Da Information - jenseits des umgangssprachlichen Gebrauchs - im Erwerbsarbeitsprozess bezeichnet, was Unklarheiten beim Erfüllen eines konkreten Arbeitsauftrags beseitigt, ist beim Ermitteln des Informationsbedarfs in Erwerbsarbeitsprozessen zu bestimmen, was diese Unklarheiten sind und was sie behebt.

Das ist nicht identisch mit dem Datenüberschuss, der die umgangssprachliche Informationsflut, Informationsüberlastung etc. erzeugt.

Es geht dabei auch nicht um das Aufspüren möglicher Wissenslücken, sondern um Defizite in der aktuellen Kommunikation zum konkreten Arbeitsprozess.

Ein kognitionswissenschaftlich begründetes (z. B. Hacker 2005; Hacker und Sachse 2014) und praktisch bewährtes Hilfsmittel wird umgangssprachlich als W-Fragen-Konzept bezeichnet. Es baut auf dem Konzept des Handlungsschemas mit seinen „Leerstellen“ (slots; Schank 1982) auf, die die W-Fragen füllen. Es wurde gezeigt (Hacker et al. 1991), dass dieses Konzept anderen, z. B. dem GOMS-Konzept (Card et al. 1983), bei der Ermittlung des Informationsbedarfs überlegen ist.

Damit wird ermittelt:

- ob überhaupt Informationsbedarf besteht (ob = whether),

- wer Unklarheiten/Informationsbedarf hat,

- was ihm/ihr unbekannt ist,

- wofür der Nutzer diese Information benötigt,

- warum die Unklarheit/der Informationsbedarf vorliegt (z.B. weil der Nutzer auf die Information des Gebers angewiesen ist und der Informationsfluss gestört ist?),

- wann die Unklarheit behoben werden muss (z.B. um nicht zu stören),

- in welcher Beschaffenheit (begrifflich, bildlich, etc.) die Information benötigt wird.

Keine dieser W-Fragen ist überflüssig: Um für den Arbeitsprozess nützliche Information zu geben, muss klar sein, wann welche warum wozu in welcher Form benötigt wird.

Die Schwierigkeit besteht darin, dass die jeweiligen Informationsgeber zutreffend wissen - nicht vermuten müssten, welche Information der Nutzer wann etc. benötigt. Tatsächlich besteht aber häufig nur begrenzt Einblick in seinen aktuellen Informationsbedarf.

Durch geeignete Gestaltung der Arbeitsorganisation können diese verlustträchtigen Mängel im Informationsfluss verringert werden: Beispielsweise durch regelmäßige Arbeitsplatzwechsel (Arbeitsrotation) zum Wissensaufbau über die Anforderungen an anderer Arbeitsplätzen als Voraussetzung wechselseitiger Unterstützung oder Vertretung - nicht nur bei Krankheit oder Urlaub, sondern auch, um ein Kurzpausensystem ohne Arbeitsausfall zu ermöglichen.
Weitere Möglichkeiten des Informationsmanagements zum Verringern des Fehlens handlungsregulierender Information sind - außer den erwähnten Recherchen und Untersuchungen - Verbesserungen der tätigkeitsbezogenen Kommunikation in regelmäßigen lern/kompetenzfördernden Arbeitsberatungen und moderierten Gruppendiskussionen (Pietzcker und Looks 2010; Hacker 2018) der informationell verknüpften Arbeitenden.

Die praktische Konsequenz ist:

Die Informationsbedarfsermittlung, geleitet durch das W-Fragen-System als Hilfsmittel, ist ein unerlässlicher Teil der leistungs-, lern- und gesundheitsfördernden (,,menschengerechten") Arbeitsgestaltung, wie in der DIN EN ISO 6385 (2016) sowie der Gefährdungsbeurteilung gefordert.

Die Informationsbedarfsermittlung kann das Zusammentragen der Erfahrungen mehrerer Beschäftigter und deren Abstimmung in einem zeitweiligen moderierten Kleingruppenprozess (,Qualitätszirkel“) erfordern (vgl. Pietzcker und Looks 2010).

Das Ergebnis der Informationsbedarfsermittlung sollte dokumentiert werden, um als Weiterbildungsgrundlage in Arbeitsbesprechungen in Erinnerung gebracht zu werden und als Einarbeitungshilfe bei Neubesetzungen verfügbar zu sein.

\section{Gestalten gebrauchstauglicher Information}

Die o.g. Frage in welcher Form eine Information benötigt wird, ist zu vertiefen. Sie führt zur Gebrauchstauglichkeit in Anlehnung an DIN EN ISO 9241-11 (2018).

Die Forderung nach Gebrauchstauglichkeit der Information bedeutet, dass nicht nur irgendwie benötigte Informationen - anstatt aufgabenirrelevanter Daten - geboten werden sollen, sondern, dass sie die für die Handlungsregulation erforderliche Darstellung haben muss, um Übersetzungsaufwand zu sparen (zur psychischen Regulation von Handlungen, vgl. Hacker 2015).

Nicht gebrauchstaugliche Information benötigt zusätzlich Zeit und mentalen Aufwand für das Umformen in eine handlungsregulierende Form und kann damit zum Erleben von beanspruchender Informationsüberlastung und zur Leistungsminderung beitragen.

$\mathrm{Zu}$ prüfen und erforderlichenfalls zu korrigieren ist

- zeitliche Passung, d.h. Informationszuführung zum Bedarfszeitpunkt (nicht zu spät, aber auch nicht zu früh (sonst Störung bei anderer Tätigkeit; Behaltens- und Erinnerungsaufwand)),

- Vollständigkeit, 
- kein Umformungsbedarf (begrifflich in bildlich; Fachchinesisch in beherrschte Sprache; fremde in betriebsrelevante Maßeinheiten),

- klare Zuordnung zum Bedarf (z. B. Betreff-Angabe),

- Reihenfolge der Angaben gemäß der erforderlichen Bearbeitungsreihenfolge,

- keine redundante (weitschweifige) Darstellung.

Die praktische Konsequenz ist:

Es genügt nicht, die benötigte Information - anstatt aufgabenirrelevanter Daten - zu bieten. Sie sollte auch handlungsleitend sein, d.h. Zeitverluste und zusätzliche Beanspruchung vermeiden.

Eine Hilfe dafür können die obigen sechs Prüfmerkmale sein.

\section{Informationsfluss-Management in der digitalen Transformation}

Betriebliches Informationsmanagement muss unterschieden werden vom betrieblichen Wissensmanagement:

Das Informationsmanagement betrifft den Austausch von Information beim Kommunizieren zu gegenwärtig bearbeiteten Aufträgen (Mark 2002).

Wissen ist langzeitiger Gedächtnisbesitz. Handlungsregulierendes Wissen von Mitarbeitern ist das wertvolle Wissenskapital von Betrieben. Es soll durch das Wissensmanagement erhalten, ausgetauscht, vermehrt und genutzt werden - ob es dabei um sprachlich ausgesagtes (und damit digitalisierbares) oder um unbewusstes, aber im Handeln eingesetztes schweigendes Wissen geht, das durch Vormachen weitergegeben werden kann (Evans und Frankish 2009; Hacker 1992; Krause 2000).

Beim Management des Informationsflusses ist zunächst zu sichern, dass tatsächlich erforderliche Information angeboten wird und zwar in handlungswirksamer Darstellung:

\subsection{Fluss von handlungsleitender (gebrauchstauglicher) Information anstatt irrelevanter Daten - Voraussetzung der Reduktion erlebter Überlastung}

Der Abschn. 3 klärte den Unterschied zwischen Information, die Unsicherheit/Unwissen beim Bearbeiten des konkreten Arbeitsauftrages beseitigt, und auftragsirrelevanten Daten.

Das W-Fragen-Konzept (Krause 2000, zu kognitionswissenschaftlichen Grundlagen) (Abschn. 4) ist eine Checkliste zum Ermitteln handlungsleitender Information.

Nicht jede benötigte Information ist handlungsleitend, d.h. gebrauchstauglich (DIN EN ISO 9241-11 (2018)) dargestellt.
Abschn. 5 stellte Merkmale gebrauchstauglicher, d.h. handlungsleitender Information zusammen, die als Checkliste nutzbar sind.

\subsection{Rationeller Informationsfluss durch geeignete Arbeitsorganisation - Wege zur Reduktion erlebter Informationsüberlastung}

Die Vernetzung digitaler Arbeitsmittel verändert nicht selten die Arbeitsorganisation, ohne dass alle Folgen bedacht sind. Die Informations- bzw. Datenflüsse können sich dabei vermehren ohne Mehrwert zu erzeugen:

- Die Art der Arbeitsteilung bzw. Arbeitskombination bestimmt den erforderlichen Informationsfluss und damit das mögliche Erleben von Informationsüberlastung. Je stärker die Arbeitsteilung als Artteilung (Teilung zwischen Tätigkeitsarten wie Vorbereiten, Organisieren, Ausführen, Kontrollieren) ist (zu Art- vs. Mengenteilung als Formen der Arbeitsteilung vgl. Abschn. 5.2, in Hacker und Sachse 2014), desto umfangreicher ist der erforderliche Abstimmungs-/Kommunikationsbedarf, d.h. der Informationsfluss. Da die Informationsgeber nicht notwendigerweise den Informationsbedarf der Empfänger kennen, sind Zusatzaufwände mit vermehrtem Informationsaustausch möglich.

Die Reduktion überzogener Arbeitsteilung durch Arbeitserweiterung; Arbeitsbereicherung oder Arbeitsrotation reduziert den organisationsbedingten überflüssigen Informationsfluss (Hacker 2008).

- Die Fragmentierung der Arbeit beim Multi-Tasking, bei Projektorganisation oder Servicekonzepten durch Wechsel zwischen zu bearbeitenden Aufgaben und dadurch Störungen mit dem Vergessen und Wiedereinarbeiten verursachen Zeitverluste und können auch zum Eindruck von Informationsüberlastung beitragen (Czerwinski et al. 2004; Eyrolle und Cellier 2000).

Der Wechsel zwischen Aufgaben (Agilität) hat Kosten.

- Mit der Vernetzung digitaler Arbeitsmittel wächst die Anzahl der Personen, die mit einer Aufgabe in Beziehung gebracht werden. Mit der Anzahl der Beteiligten nimmt jedoch die Effizienz der Kommunikation ab (Ringelmann-Effekt; Zysno 1998), sofern sie nicht sachkundig moderiert wird (Pietzcker und Looks 2010; Hacker 2018).

Das Festlegen eindeutiger Zuständigkeit und Verantwortung reduziert überflüssigen Informationsfluss.

Praktisch folgt:

Durch unzweckmäßige oder mangelhafte Organisationsgestaltung kann Daten- und auch Informationsfluss erzeugt werden, der zu Zeitverlusten und Fehlermöglichkeiten führt, die durch Reorganisation zu beheben sind (Zijlstra et al. 1999). 


\subsection{Wahl des geeigneten Mediums}

Nicht jede Information muss schriftlich ausgetauscht werden, weil digitale Arbeitsmittel (z.B. E-Mail) verfügbar sind.

Es gibt gewichtige Gründe, herkömmliche, analoge Informationsvermittlung durch Zweiergespräche von Angesicht zu Angesicht oder per Telefon sowie Arbeitsbesprechungen mit physischer Anwesenheit der Arbeitsgruppenmitglieder beizubehalten und auch nicht durch SmartPhone-Ablenkungen unterlaufen $\mathrm{zu}$ lassen (Schroeder 2014; Wolf 2018):

- im Face-to-Face Gespräch ist der Partner zeitgleich als Mensch - nicht nur als E-Mail-Adresse - gegenwärtig;

- Sehen und Hören, Aussage und Ausdruck (Mimik, Gestik, Sprach-Melos) sind wirksam zur Informationsübermittlung;

- ein Wechselgespräch anstatt zeitlich versetzten Textaustauschs findet statt;

- damit wird bei Bedarf Gemeinsamkeit im Prozess der Ideen-Entwicklung und Lösungsentwicklung unterstützt (,,unsere Lösung");

- Missverständnisse werden im Entstehen erkennbar und damit vermeidbar;

- gesprochene Sprache ist für die Mehrzahl der Menschen leichter verständlich und informativer als Schriftsprache.

Arbeitsbesprechungen vor Ort ggf. mit Demonstrationen, Vor- und Nachmachen von Handlungen haben größeren Lern-/Erinnerungseffekt als digitale Darstellungen allein. Am größten ist der Lerneffekt beim Veranlassen zu handschriftlichen anstatt tastatur-vermittelten, ,eingetippten" Notizen (Mueller und Oppenheimer 2014).

Die Möglichkeit der Medienwahl sollte bewusst genutzt und bezogen auf eine menschengerechte Arbeitsgestaltung überdacht werden. Auch darin besteht eine Möglichkeit, mögliche Informationsüberlastung bei der Arbeit mit digitalen Arbeitsmitteln zu reduzieren.

\subsection{Wahl anforderungsgerechter informationstechnischer Systeme}

Das Auswählen oder Gestalten von gebrauchstauglichen informationstechnischen Systemen (Hard- und Software) unterstützt die DIN EN ISO 9241-11 (2018) mit differenzierten praktischen Hinweisen.

Bei der Auswahl von informationstechnischen Systemen für die digitale Transformation sind neben informationstechnischen auch inhaltliche Fragen zu berücksichtigen: Leisten diese Systeme
- technische Unterstützung beim Ausschluss nicht arbeitsbezogener, privater Kommunikation während der $\mathrm{Ar}$ beitszeit;

- technische Unterstützung beim Reduzieren von für den jeweiligen Adressaten auftragsirrelevanten Daten hinausgehend über das Ausfiltern von Spam;

- technische Unterstützung bei gezielten anstatt breit gestreuten Adressatenfestlegungen im Interesse des Vermeidens z.B. von überflüssigen CC-Setzungen (Mark 2002);

- technische Unterstützung beim Einhalten gewünschter störungsfreier (z.B. E-Mail-freier) Arbeitszeiten von Adressaten (Mark et al. 2008);

- ein Angebot optimierter (Text)Bausteine für einen gebrauchstauglichen Informationsfluss zu wiederkehrenden Inhalten;

- die Wahl zwischen automatischer versus menschengestützter Ausführung von Arbeitsprozessen/-schritten durch die Arbeitenden (z.B. zum Erhalten ihrer Qualifikation für den Fall von Systemstörungen) (Wolf 2018)?

\subsection{Generationsunterschiede im Informationsmanagement}

Der hohe Anteil Erwerbstätiger in der zweiten Hälfte des Erwerbslebensalters sowie der derzeit wirksame Generationseffekt bei der Vertrautheit mit digitalen Hilfsmitteln und künstlicher Intelligenz stellt Anforderungen auch an das betriebliche Informationsmanagement.

Der objektive Bedarf an auftragsbezogener Information und das Erfordernis ihrer handlungsgerechten, gebrauchstauglichen Darstellung ist bei anforderungsgerechter Ausund Weiterbildung für unterschiedliche Generationen von Erwerbstätigen gleich.

Unterschiede sind nach derzeitigem Wissen zu erwarten bei der Bevorzugung der Medien des Informationsangebots zwischen den jüngeren digital natives und den älteren digital immigrants. Die letzteren dürften arbeitslebenslang vorwiegend mit analogen Medien gearbeitet haben und diese bevorzugen (Zacher et al. 2016).

Zum Vorliegen und zur Ausprägung dieser Tendenz fehlen noch belastbare branchen- und tätigkeitsspezifische Befunde. Insbesondere dürfte die Ausprägung dieser Tendenz abhängig und damit beeinflussbar sein von der tätigkeitsbegleitenden Weiterbildung im Sinne des learning on the job (Salas et al. 2012).

\section{Fazit}

Die dargestellten Aspekte des Informationsmanagements und ihre jeweiligen Einzelmerkmale skizzieren ein ausbau- 
fähiges System von Hilfen für das Bewerten und das Gestalten des Informationsflusses in Unternehmen im Interesse sowohl der Arbeitsleistung als auch des Vermeidens von informationeller Fehlbeanspruchung der Arbeitenden.

\section{Funding Open Access funding provided by Projekt DEAL.}

Open Access Dieser Artikel wird unter der Creative Commons Namensnennung 4.0 International Lizenz veröffentlicht, welche die Nutzung, Vervielfältigung, Bearbeitung, Verbreitung und Wiedergabe in jeglichem Medium und Format erlaubt, sofern Sie den/die ursprünglichen Autor(en) und die Quelle ordnungsgemäß nennen, einen Link zur Creative Commons Lizenz beifügen und angeben, ob Änderungen vorgenommen wurden.

Die in diesem Artikel enthaltenen Bilder und sonstiges Drittmaterial unterliegen ebenfalls der genannten Creative Commons Lizenz, sofern sich aus der Abbildungslegende nichts anderes ergibt. Sofern das betreffende Material nicht unter der genannten Creative Commons Lizenz steht und die betreffende Handlung nicht nach gesetzlichen Vorschriften erlaubt ist, ist für die oben aufgeführten Weiterverwendungen des Materials die Einwilligung des jeweiligen Rechteinhabers einzuholen.

Weitere Details zur Lizenz entnehmen Sie bitte der Lizenzinformation auf http://creativecommons.org/licenses/by/4.0/deed.de.

\section{Literatur}

Attneave FA (1965) Informationstheorie in der Psychologie. Huber, Bern, Stuttgart

Baethge A, Rigotti T (2013) Interruptions to workflow: their relationship with irritation and satisfaction with performance, and the mediating roles of time pressure and mental demands. Work Stress 27(1):43-63

Card SK, Moran TP, Newell A (1983) The psychology of human-computer-interaction. Erlbaum, Hillsdale

Cowan N (2010) The magical mystery four: how is working memory capacity limited, and why? Curr Dir Psychol Sci 19(1):51-57

Czerwinski M, Bratic M, Horvitz E, Wilhite S (2004) A diary study of task switching and interruptions. CHI Conf Proc 6(1):175-182

DIN EN ISO 6385 (2016) Grundsätze der Ergonomie für die Gestaltung von Arbeitssystemen. Berlin, Beuth

DIN EN ISO 9241-11 (2018) Gestaltung gebrauchstauglicher interaktiver Systeme. Berlin, Beuth

Drössler S, Steputat A, Schubert M, Günther N, Staudte R, Kofahl M, Hegewald J, Seidler A (2018) Informationsüberflutung durch digitale Medien am Arbeitsplatz. Zentralbl Arbeitsmed Arbeitsschutz Ergonomie 68(2):77-88

Evans JS, Frankish K (Hrsg) (2009) In two minds: dual process and beyond. Oxford University Press, New York

Eyrolle H, Cellier JM (2000) The effects of interruptions in work activity: field and laboratory results. Appl Ergon 31(5):537-453

Hacker W (1992) Expertenkönnen - Erkennen und Vermitteln. Verlag für Angewandte Psychologie, Göttingen, Stuttgart

Hacker W (2005) Allgemeine Arbeitspsychologie. Huber Hogrefe, Bern

Hacker W (2008) Informationsflussgestaltung als Arbeits- und Organisationsoptimierung. Reihe MTO, Bd. 44. vdf Hochschulverlag der ETH, Zürich

Hacker W (2015) Psychische Regulation von Arbeitstätigkeiten. Kröning, Asanger
Hacker W (2018) Kooperative Arbeitsgestaltung durch Arbeitsplatzinhaber-/innen - ein Lernprozess. Arbeit 27(4):391-400

Hacker W (2020) Prävention von zeitlicher Überforderung bei entgrenzter Wissens- sowie Innovationsarbeit: Möglichkeiten und Grenzen der Zeitbedarfsermittlung - eine Fallstudie. J Psychol Alltagsh 13(1):12-27

Hacker W, Sachse P (2014) Allgemeine Arbeitspsychologie - Psychische Regulation von Tätigkeiten. Hogrefe, Göttingen

Hacker W, Großmann N, Teske-El Kodwa S (1991) Knowledge elicitation: a comparison of models and methods. In: Bullinger HJ (Hrsg) Human aspects in computing. Elsevier, Amsterdam, S 861-865

Hassin RR (2014) Mining the unconscious. Psychological science digs into the power of nonconscious processing. APS Observ 24(4): $12-36$

Junghanns G, Kersten N (2020) Informationsüberflutung am Arbeitsplatz - gesundheitliche Konsequenzen. Zentralbl Arbeitsmed Arbeitsschutz Ergonomie 70:8-17

Krause W (2000) Denken und Gedächtnis aus naturwissenschaftlicher Sicht. Hogrefe, Göttingen

Kuhlmann M (2002) Das Beobachtungsinterview als Methode der Organisationsforschung. In: Kühl S, Strodholz P (Hrsg) Methoden der Organisationsforschung. Rowohlt, Reinbeck

Lin BC, Kain JM, Fritz C (2013) Don't interrupt me! An examination of the relationship between intrusions at work and employee strain. Int J Stress Manag 20(2):77-94

Mark G (2002) Extreme collaboration. Commun ACM 45(6):89-93

Mark G, Gudith D, Klocke U (2008) The cost of interrupted work: more speed, more stress. In: Burnett M, Costabile MF, Catarei T, de Ruyter B, Tan D, Czerwinski M, Lund A (Hrsg) Proceedings of the SIGCHI conference on human factors in computing systems (CHI 2008). ACM Press, New York, S 107-110

Mueller PA, Oppenheimer DM (2014) The pen is mightier than the keyboard. Advantages of longhand over laptop note taking. Psychol Sci 25(6):1159-1168

Pietzcker F, Looks P (Hrsg) (2010) Der Aufgabenbezogene Informationsaustausch - zeitweilige partizipative Gruppenarbeit zur Problemlösung. Reihe MTO, Bd. 45. vdf Hochschulverlag der ETH, Zürich

Rau R, Göllner C (2018) Rahmenmodell der Arbeitsintensität als objektiv bestehende Anforderung. Arbeit 27(2):151-174

Salas E, Tannenbaum SJ, Kraiger K, Smith-Jentsch KA (2012) The science of training and development in organizations: what matters in practice. Psychol Sci Public Interest 13(2):73-96

Schank RO (1982) Dynamic memory. University Press, Cambridge

Schroeder S (2014) From principles of cognitive science to moods. APS Observ 27(6):25-26

Seidler A, Steputat A, Drössler S, Schubert M, Günther N, Staudte R, Kofahl M, Hegewald J (2018) Determinanten und Auswirkungen von Informationsüberflutung am Arbeitsplatz. Ein systematisches Review. Zentralbl Arbeitsmed Arbeitsschutz Ergonomie 68(1):12-26

Wolf M (2018) Reader come home. The reading brain in a digital world. Harper, New York

Zacher H, Hacker W, Frese M (2016) Action regulation across the lifespan (ARAL): a meta-theory of work and aging. Work Aging Retire 2(3):286-306

Zijlstra FR, Roe RA, Leonora AB, Krediet I (1999) Temporal factors in mental work. Effects of interrupted activities. J Occup Organ Psychol 72(2): 163-185

Zysno P (1998) Vom Seilzug zum Brainstorming. Die Effizienz der Gruppe. In: Witte EH (Hrsg) Sozialpsychologie der Gruppenleistung. Pabst, Lengerich, S 184-220 